\title{
Plasmacytoid Melanoma of the Urinary Bladder and Lymph Nodes with Immunohistochemical Expression of Plasma Cell Markers Revealing Primary Esophageal Melanoma
}

\author{
Slim Charfi, ${ }^{1}$ Sameh Ellouze, ${ }^{1}$ Hela Mnif, ${ }^{1}$ Ali Amouri, ${ }^{2}$ \\ Abdelmajid Khabir, ${ }^{1}$ and Tahya Sellami-Boudawara ${ }^{1}$ \\ ${ }^{1}$ Department of Pathology, CHU Habib Bourguiba, CP 3029, Sfax, Tunisia \\ ${ }^{2}$ Department of Gastroenterology, CHU Hedi Chaker, CP 3029, Sfax, Tunisia \\ Correspondence should be addressed to Slim Charfi, charfislim@gmail.com \\ Received 31 July 2012; Accepted 1 October 2012 \\ Academic Editors: M. Marino and Z. Schaff
}

Copyright ( $) 2012$ Slim Charfi et al. This is an open access article distributed under the Creative Commons Attribution License, which permits unrestricted use, distribution, and reproduction in any medium, provided the original work is properly cited.

Plasmacytoid variant of melanoma is reported in only rare cases. We present the case of a 54-years-old man admitted for enlarged lymph nodes in the lumbar region. Initial diagnosis of plasmablastic lymphoma/plasma cell myeloma was considered. At our institute, a bladder polyp was removed. Microscopic exam demonstrated dense plasmacytoid cells infiltration with pigment deposits. Immunohistochemical study showed strong expression of HMB45, Melan A, and vimentin. There was focal positivity with S100 protein and CD138/syndecan-1. The diagnosis of metastatic plasmacytoid melanoma was finally established. Clinical exam revealed an esophageal melanoma with melanosis supporting its primary location. Although rarely, melanoma especially plasmacytoid variant may express plasma cell markers which may lead to erroneous diagnosis of plasma cell proliferation. Careful morphological examination for melanin pigment and the use of panel of melanocytic markers are helpful for diagnosis.

\section{Introduction}

Melanomas, particularly noncutaneous primaries and metastasis, are known to display tremendous pathological diversity which may mimic many other tumors [1]. This diversity includes cytomorphology, architecture, stromal component, and immunophenotype. Plasmacytoid variant of melanoma is reported in only rare cases $[1-5]$. Bladder metastasis of melanoma are extremely rare $[6,7]$. To our knowledge, no bladder metastasis from a primary esophageal melanoma has been previously reported.

\section{Case Presentation}

The patient is a 54 years old man, with no medical history, admitted for investigation of enlarged lymph nodes of the lumbar region with a diagnosis of plasmablastic lymphoma/plasma cell myeloma. This diagnosis was established outside our institute on CT-scan lymph node biopsy. Initial pathologist described in his report a diffuse infiltration by plasmacytoid cells with immunohistochemistry expression of CD138/syndecan-1, MUM1, and immunoglobulin lambda light chain. Tumor cells were negative for S100 protein, kappa light chain, CD3, CD20, CD79a, and keratin KL1. HMB45 and Melan A were not tested. Laboratory analysis revealed an IgG lambda monoclonal immunoglobulin at immunofixation. The patient developed an acute renal failure. Cystoscopy exam demonstrated a $0,5 \mathrm{~cm}$ sessile bladder polyp which was removed. Microscopic exam showed a diffuse, dense, plasmacytoid cellular proliferation (Figure 1). Cells were small to medium with eosinophilic cytoplasm and eccentric nuclei with central prominent nucleoli. Some cells were pigmented (Figure 2). Tumor cells were strongly and diffusely positive for HMB45, Melan A, and vimentin. They were focally positive for S100 Protein, CD138/syndecan1 , and immunoglobulin lambda light chain (Figure 3). Tumor cells were negative for keratin AE1/AE3, keratin 7, keratin 20, epithelial membrane antigen (EMA), CD79a, and immunoglobulin kappa light chain (Table 1). MUM1 was not available at our department. Thus, the diagnosis 


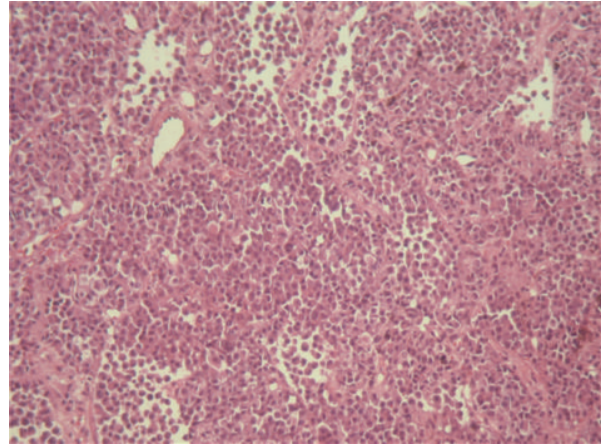

FIGURE 1: Diffuse proliferation of rounded neoplastic cells showing incohesion $(\mathrm{HE} \times 40)$.

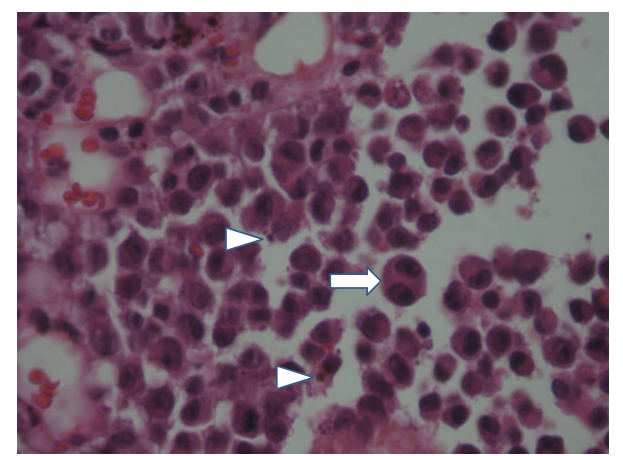

FIGURE 2: neoplastic cells demonstrated eosinophilic cytoplasm and eccentric nuclei with prominent nucleoli; some cells are binucleated $(\Rightarrow)$; note the presence of pigment $(\triangleright)(\mathrm{HE} \times 400)$.

was redressed to metastatic plasmacytoid melanoma. Microscopic examination of bone morrow was unremarkable. The patient underwent an upper endoscopy, which revealed a $2 \mathrm{~cm}$, lobulated, and pigmented mass located in the junction medium-distal esophagus. Biopsy of this mass demonstrated a tumor proliferation containing a mixture of epithelioid and spindle-shaped cells arranged in fascicles with presence of melanin pigment (Figure 4). There were some cells with plasmacytoid feature (Figure 5). Immunohistochemically, tumor cells were positive for HMB45, Melan A, S100 protein, and only focally for CD138/syndecan-1. HMB45 staining showed an increased number of melanocytes at the basal layer of the squamous epithelium (Figure 6) suggesting the presence of melanosis and furthermore that this location represents the primary melanoma. The patient died one month after the final diagnosis.

\section{Discussion}

Plasmacytoid variant of melanoma is a rare finding [1$5,8]$ which may mimic many other entities especially plasma cell proliferation. The use of immunohistochemistry in the diagnosis of such tumors is primordial. However, as shown in our case, this study may lead to erroneous diagnosis. In fact, we observed a positive staining in primary oesophageal and metastasis melanoma with the plasma cell
TABLE 1: Panel of immunohistochemical stains.

\begin{tabular}{lccc}
\hline Antibody & Clone & Company & Dilution \\
\hline Keratin & AE1/AE3 & DAKO & $1: 50$ \\
CD138 & MI15 & DAKO & $1: 50$ \\
MelanA & A103 & DAKO & $1: 50$ \\
Vimentin & V9 & DAKO & $1: 50$ \\
HMB45 & HMB45 & DAKO & $1: 50$ \\
Lambda immunoglobulin & A0193 & DAKO & $1: 2000$ \\
light chain & & & \\
Kappa immunoglobulin & A0191 & DAKO & $1: 2000$ \\
light chain & & & \\
Keratin 7 & OV-TL 12/30 & DAKO & $1: 50$ \\
Keratin 20 & Ks20.8 & DAKO & $1: 50$ \\
EMA & E29 & DAKO & $1: 100$ \\
CD79a & JCB117 & DAKO & $1: 50$ \\
\hline
\end{tabular}

markers CD138/syndecan-1, MUM1, and immunoglobulin lambda light chain. The expression of CD138/syndecan1 in melanoma is reported in only one case [8]. It consists of a large ulcerated cutaneous melanoma which was initially considered as extramedullary plasmocytoma. CD138/syndecan-1 is a heparin sulphate bearing proteoglycan. CD138/syndecan-1 plays an important role in plasma cell differentiation and functions in adhesion and motility of cells. It is expressed on most of the myeloma tumour cells and cells of certain other tumors of $b$ lineage and also on epithelial cells [9]. MUM1 is a member of the interferon regulatory factor family of transcription factors. In hematolymphoid system, MUM1 plays a significant role in terminal B-cell differentiation and hence is a potentially specific marker for plasmacytic differentiation [10]. MUM1 is present in a wide spectrum of hematolymphoid neoplasms and in malignant melanomas but is absent in the other human neoplasms $[10,11]$. The study of Sundram et al. has shown that MUM1 is more sensitive than both HMB45 and Melan A in cases of conventional primary and metastatic melanomas. MUM1 was also positive for some cases that were weakly positive with S100 protein [10]. Shanks and Banerjee reported the expression of another plasma cell marker VS38 in melanoma [12]. The immunohistochemical expression of lambda light chain in our case was associated to the detection of IgG lambda monoclonal immunoglobulin in serum immunofixation. Immunoglobulin light chain restriction by flow cytometry of tumor cells was also reported by Lehmer et al. [8]. The signification of the expression of plasma cell markers and their role in the biologic behaviour of melanocytic tumors are unclear and may need additional studies for clarification. We think that these findings represent a probably aberration in immunophenotypic melanoma. Lehmer el al. suggested that neoplastic plasma cells are associated to melanoma participating in its chronic inflammatory [8]. Immunohistochemical aberrant findings in melanoma are well known. The most frequent of these is cytokeratin which may expressed up to $10 \%$ in melanoma. Expression of neuroendocrine markers has also been reported $[1,5]$. 


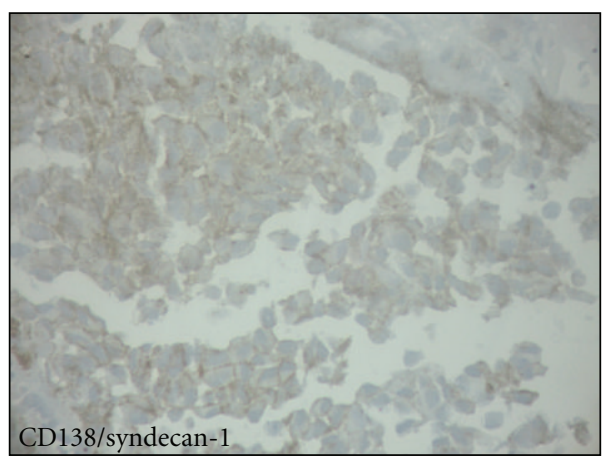

(a)

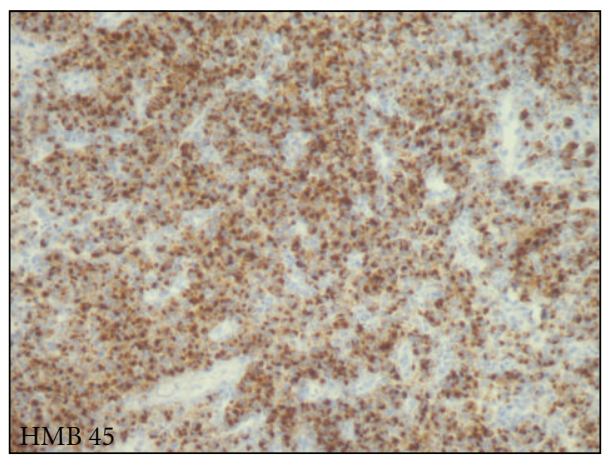

(c)

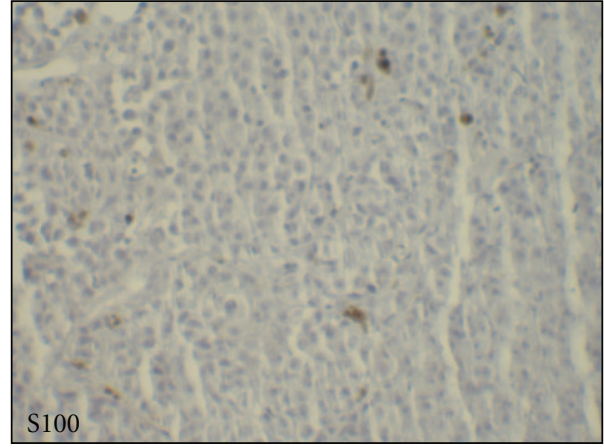

(b)

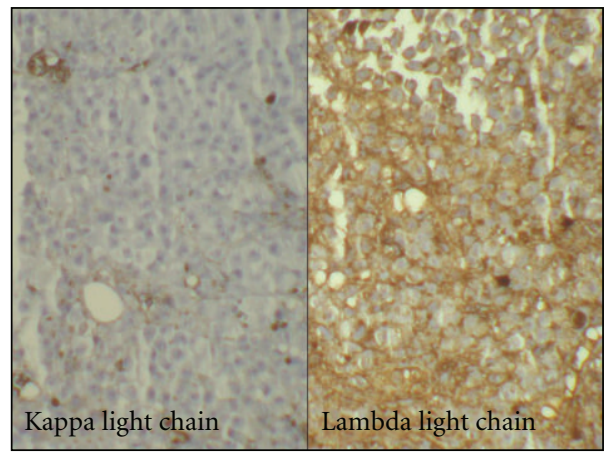

(d)

FIGURE 3: (a) Neoplastic cells exhibit immunoreactivity for CD138 $(\times 100)$. (b) Very rare neoplastic cells are positive for S100 protein $(\times 100)$. (c) Strong HMB45 expression by the neoplastic cells $(\times 40)$. (d) Neoplastic cells are positive for lambda light chain and negative for kappa light chain $(\times 100)$.

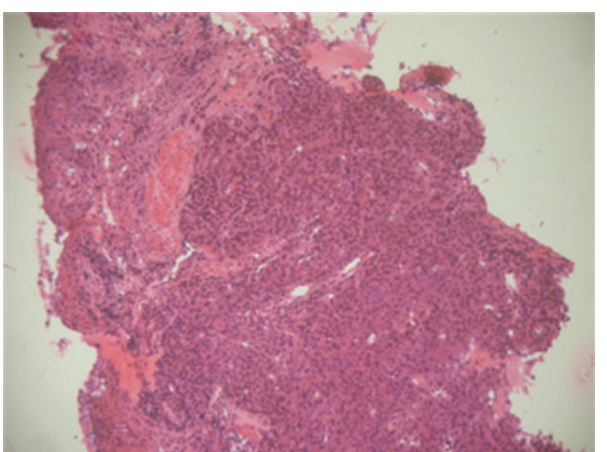

FIGURE 4: Esophageal tumor containing a mixture of epitheliod and spindle-shaped cells $(\mathrm{HE} \times 40)$.

Our case showed an immunophenotypic heterogeneity of S100 protein expression between primary esophageal and metastasis locations. S100 protein is expressed in $94 \%$ and $95 \%$ of primary and metastatic melanomas, respectively [13]. Only 3-4\% of melanomas were S100 protein negative. Morphologically, these melanomas present as in our case are atypical features (signet cell, rhabdoid, etc.) $[1,5]$.

Bladder metastasis from melanoma is a very rare finding. Less than 10 cases were reported in the English literature. Cutaneous melanoma represents most primary location $[6$, 7]. To our knowledge, bladder metastasis from primary esophageal melanoma was not previously reported.

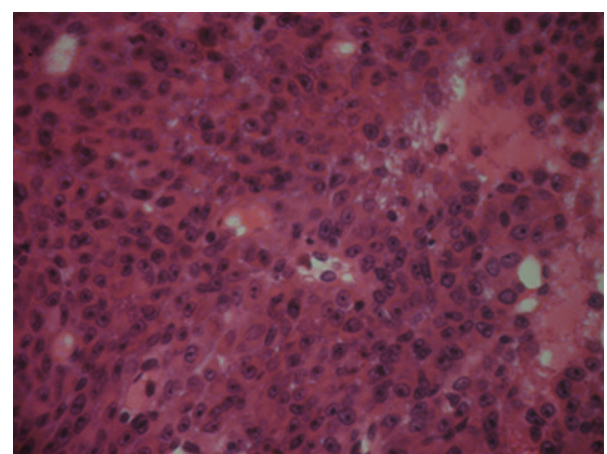

FIGURE 5: Esophageal tumor-sheets of large cells with plasmacytoid features $(\mathrm{HE} \times 400)$.

Primary esophageal melanoma represents 0.1 to $0.5 \%$ of the primary malignant esophageal neoplasms and approximately $0.5 \%$ of melanoma originate in the esophagus. Cutaneous malignant melanomas metastatic to the esophageal are more common than primary esophageal melanoma [14]. The histopathologic criteria for diagnosing primary esophageal melanoma have not been clearly established. Sanchez et al. reported that in situ melanoma is the most consistent criterion. However, this criterion is rarely present, as in our cases, in mucosal biopsies [15]. Two additional criteria have been proposed by Sabanathan et al.: presence of esophageal melanocytosis and a diagnosis of exclusion. 


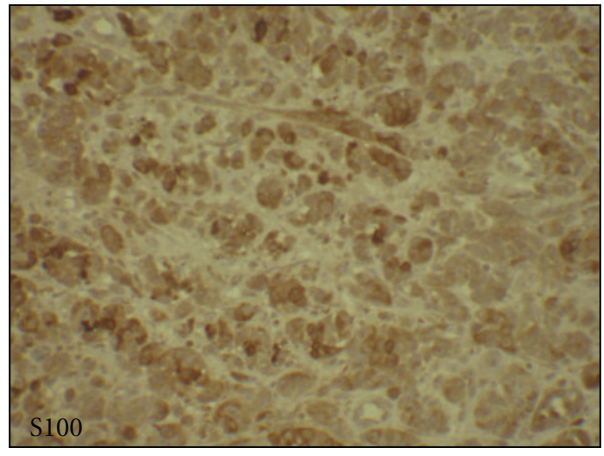

(a)

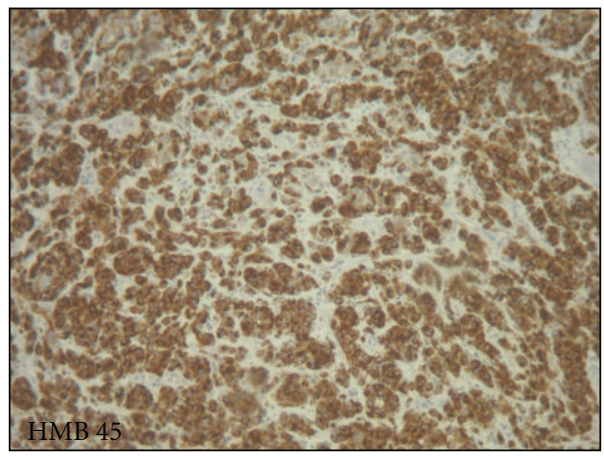

(c)

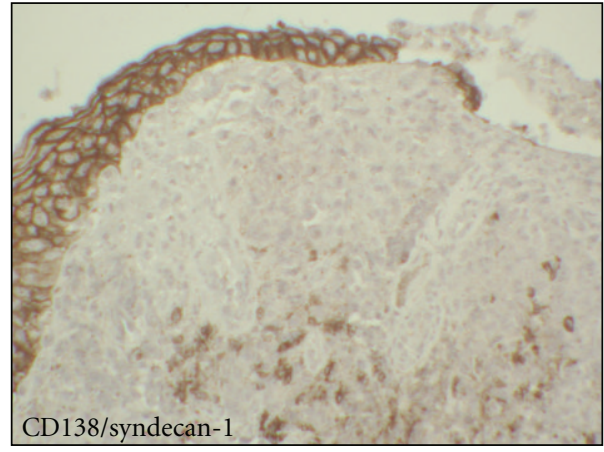

(b)

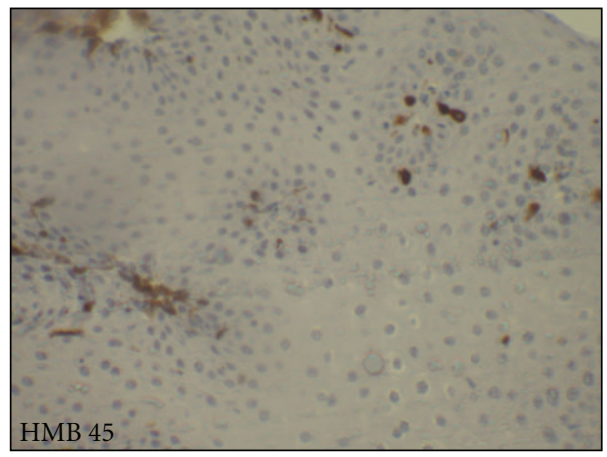

(d)

Figure 6: (a) Neoplastic cells showed strong positivity with S100 protein $(\times 100)$. (b) Focal expression for CD138/syndecan-1 ( $\times 100)$. (c) Neoplastic cells showed strong positivity with HMB45 $(\times 40)$. (d) HMB45 staining showing increased number of melanocytic cells at the basal layer of the squamous epithelium $(\times 100)$.

Melanocytosis is characterized by the presence of increased melanocytes in the basal layer of esophageal squamous mucosa, and the melanocytes do not show atypia [14].

In Conclusion, this paper emphasises that plasma cell markers are not entirely specific and are particularly expressed in melanoma. We recommend a panel of immunohistochemical markers which should include more than one melanocytic marker to exclude malignant melanoma, even if the tumour has a plasmacytoid appearance suggestive of plasma cell proliferation. In addition, careful morphological assessment and particularly the research of melanin pigment reduce the risk of erroneous interpretation of aberrant results.

\section{Conflict of Interests}

There is no conflict of interests to be declared.

\section{References}

[1] S. S. Banerjee and M. Harris, "Morphological and immunophenotypic variations in malignant melanoma," Histopathology, vol. 36, no. 5, pp. 387-402, 2000.

[2] N. Siddaraju, P. J. Yaranal, M. M. Mishra, and J. Soundararaghavan, "Fine needle aspiration cytology in recurrent amelanotic melanoma: a case report," Acta Cytologica, vol. 51, no. 5, pp. 829-832, 2007.
[3] A. V. Parwani, T. Y. Chan, S. Mathew, and S. Z. Ali, "Metastatic malignant melanoma in liver aspirate: cytomorphologic distinction from hepatocellular carcinoma," Diagnostic Cytopathology, vol. 30, no. 4, pp. 247-250, 2004.

[4] K. L. Ortega, N. S. de Araújo, F. B. de Souza, and M. H. C. G. Magalhães, "Primary malignant melanoma of the oral cavity: a case report," International Journal of Dermatology, vol. 43, no. 10, pp. 750-752, 2004.

[5] N. D. Riddle and M. M. Bui, "When melanoma is negative for S100: diagnostic pitfalls," Archives of Pathology \& Laboratory Medicine, vol. 136, pp. 237-239, 2012.

[6] O. Efesoy and S. Cayan, "Bladder metastasis of malignant melanoma: a case report and review of literature," Medical Oncology, vol. 28, supplement 1, pp. 667-669, 2010.

[7] V. Menéndez López, R. Alvarez-Vijande García, M. Solé Arqués, and G. P. Carretero, "Bladder metastasis of a malignant melanoma: case report," Archivos Espanoles de Urologia, vol. 55, no. 10, pp. 1277-1279, 2002.

[8] L. M. Lehmer, B. D. Ragsdale, M. V. Frost, and K. L. Ferguson, "Large neglected ulcerated melanoma mimicking extramedullary plasmacytoma," The American Journal of Dermatopathology, vol. 33, pp. 94-98, 2011.

[9] R. D. Sanderson and M. Børset, "Syndecan-1 in B lymphoid malignancies," Annals of Hematology, vol. 81, no. 3, pp. 125$135,2002$.

[10] U. Sundram, J. D. Harvell, R. V. Rouse, and Y. Natkunam, "Expression of the B-cell proliferation marker MUM1 by melanocytic lesions and comparison with S100, gp100 
(HMB45), and MelanA," Modern Pathology, vol. 16, no. 8, pp. 802-810, 2003.

[11] Y. Natkunam, R. A. Warnke, K. Montgomery, B. Falini, and M. van de Rijn, "Analysis of MUM1/IRF4 protein expression using tissue microarrays and immunohistochemistry," Modern Pathology, vol. 14, no. 7, pp. 686-694, 2001.

[12] J. H. Shanks and S. S. Banerjee, "VS38 immunostaining in melanocytic lesions," Journal of Clinical Pathology, vol. 49, no. 3, pp. 205-207, 1996.

[13] P. W. Bishop, L. P. Menasce, A. J. Yates, N. A. Win, and S. S. Banerjee, "An immunophenotypic survey of malignant melanomas," Histopathology, vol. 23, no. 2, pp. 159-166, 1993.

[14] S. Sabanathan, J. Eng, and G. N. Pradhan, "Primary malignant melanoma of the esophagus," American Journal of Gastroenterology, vol. 84, no. 12, pp. 1475-1481, 1989.

[15] A. A. Sanchez, T. T. Wu, V. G. Prieto, A. Rashid, S. R. Hamilton, and $\mathrm{H}$. Wang, "Comparison of primary and metastatic malignant melanoma of the esophagus: clinicopathologic review of 10 cases," Archives of Pathology and Laboratory Medicine, vol. 132, no. 10, pp. 1623-1629, 2008. 


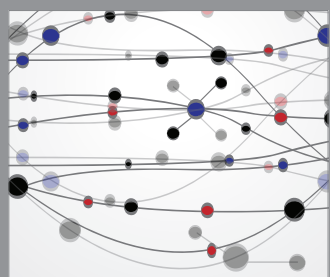

The Scientific World Journal
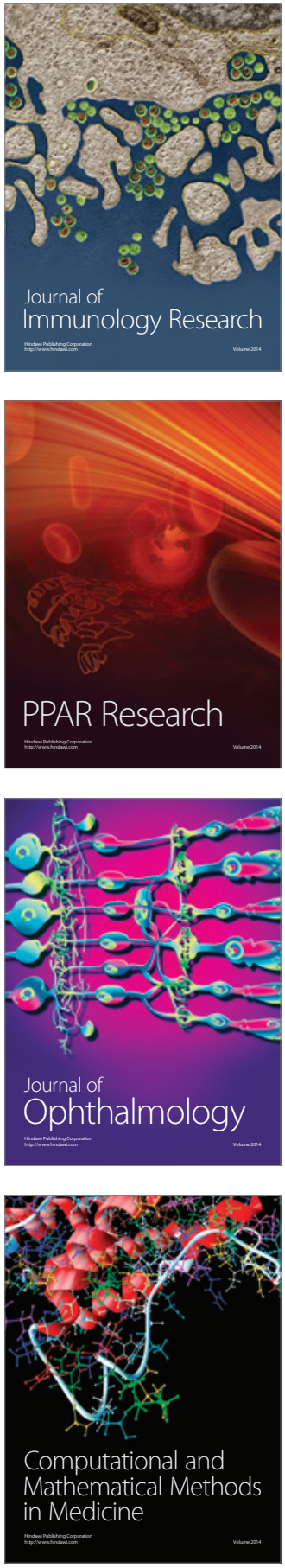

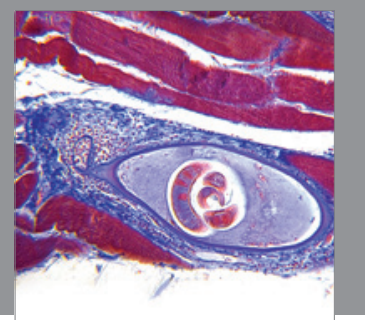

Gastroenterology

Research and Practice
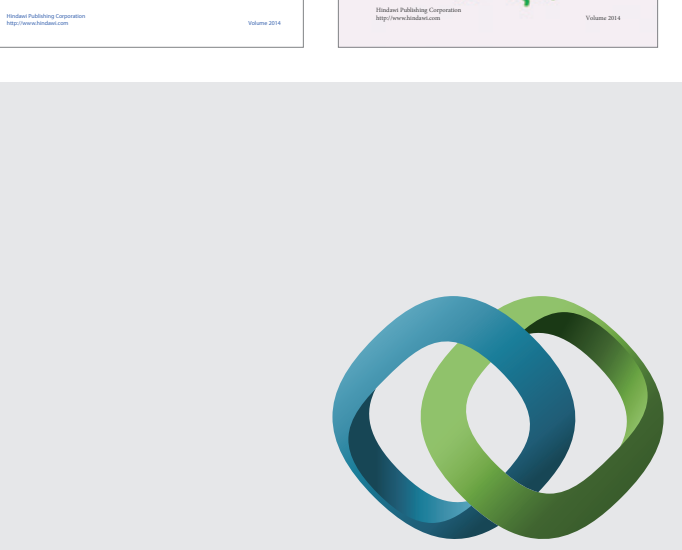

\section{Hindawi}

Submit your manuscripts at

http://www.hindawi.com
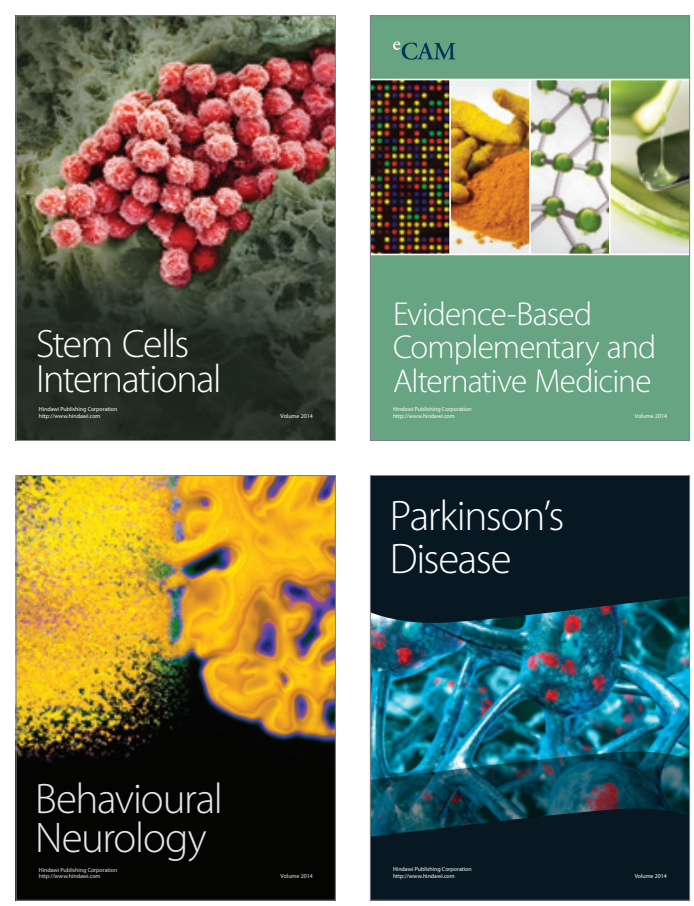

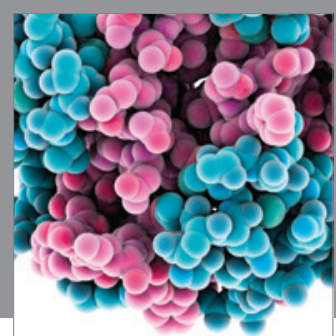

Journal of
Diabetes Research

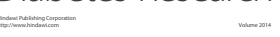

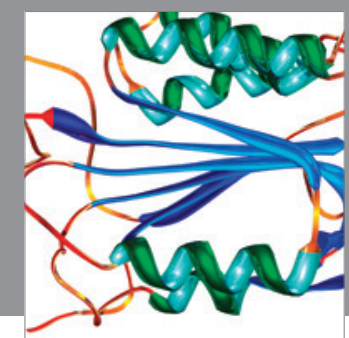

Disease Markers
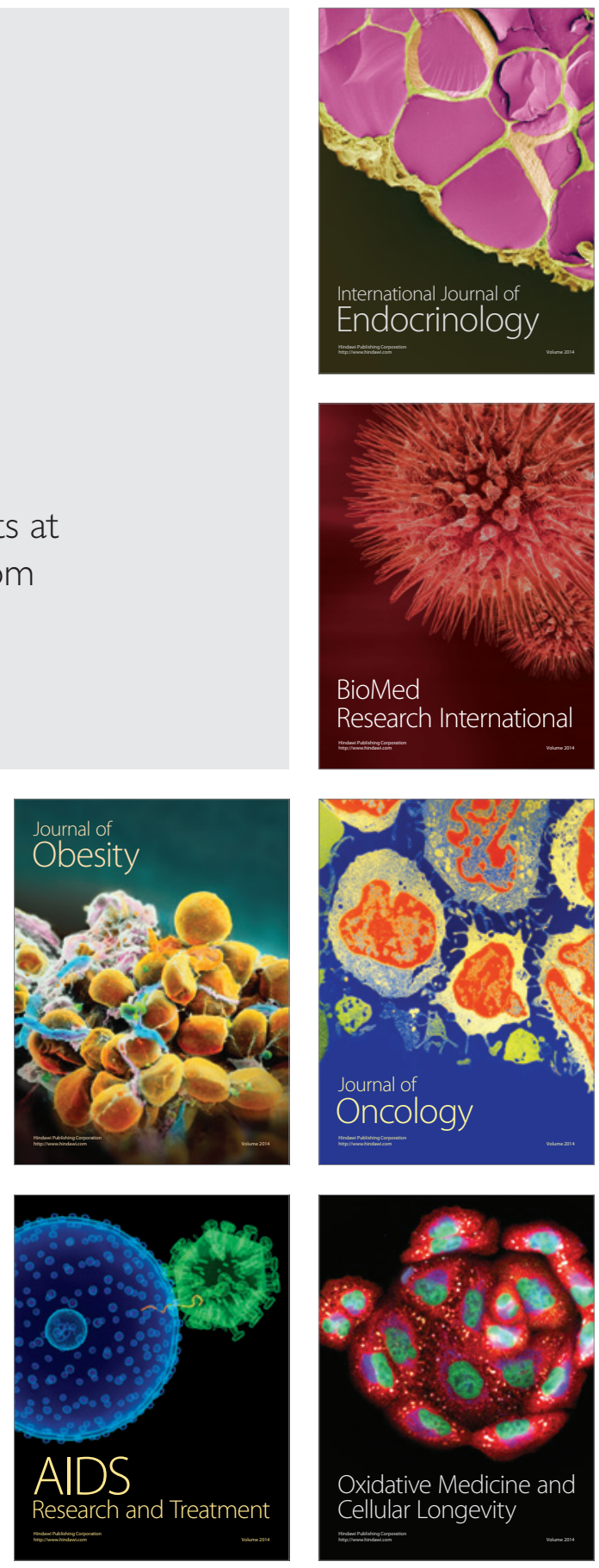